\title{
RECÉM-NASCIDOS COM REFLEXO VERMELHO "SUSPEITO": SEGUIMENTO EM CONSULTA OFTALMOLÓGICA
}

\author{
Red relex "suspect" in newborn: follow up in \\ ophtalmologic consultation
}

\section{Los recién nacidos con reflejo rojo "sospechoso": seguimiento en la consulta oftalmológica}

\author{
Maria Vera Lúcia Moreira Leitão Cardoso \\ Ingrid Martins Leite Lúcio ${ }^{3}$
}

\author{
Adriana Sousa Carvalho de Aguiar² \\ Islane Castro Verçosa ${ }^{4}$
}

\section{RESUMO}

0 teste do reflexo vermelho em recém-nascidos é uma forma de avaliação visual, permitindo a identificação precoce de leucocorias, presente frequentemente na catarata congênita, retinoblastoma e retinopatia da prematuridade. Objetivou-se avaliar, em consulta pelo oftalmologista, o resultado da avaliação pelo teste do reflexo vermelho realizado por enfermeiras, considerado "suspeito" em um grupo de recém-nascidos. Estudo descritivo, realizado em uma instituição pública, em Fortaleza-CE, de novembro/2005 a março/2006. Foi realizado por duas enfermeiras e uma aluna de enfermagem com 180 recém-nascidos; 24 destes apresentaram coloração do reflexo fora do padrão, sobressaindo-se amarelo com áreas mais esbranquiçadas ao centro ou com presença de rajas. Porém, somente foi possível encaminhamento de seis crianças para oftalmologista. Após fundoscopia, os achados encontrados no teste do reflexo vermelho foram confirmados, porém considerados variações da normalidade. Intervenções multiprofissionais favorecem o desenvolvimento de ações eficazes voltadas à prevenção da cegueira e continuidade do processo de cuidado para essas crianças.

Palavras-chave: Saúde Ocular. Cegueira. Recém-Nascido.

\begin{abstract}
The red reflex test in newborns is a visual screening way, allowing the early identification of leukokorias which are present, often on congenital cataract, on retinoblastoma and on retinopathy of prematurity. It aimed to evaluate, during ophthalmologist consultation, the suspect result of the screening through the red reflex test made by nurses in a group of newborns. Descriptive study carried out in a public institution in Fortaleza-CE, from November 2005 to March 2006. The red reflex test was performed by two nurses and one nursing student with 180 newborns; from these, 24 showed coloration of the reflex out of standard, standing out yellow color with whitish areas on center or with presence of stripe. However, it was possible to follow to ophthalmologist, only six babies. After fundoscopy, the findings detected on red reflex test made by nurses were confirmed, but considered variations of normality. Multiprofessionals interventions benefit the development of effective actions to prevention of blindness and the continuity of the care process for these babies.
\end{abstract}

Keywords: Eye Health. Blindness. Newborn.

\section{Resumen}

El examen del reflejo rojo en recién nacidos es una forma de evaluación visual, que permite la identificación precoz de leucocorias, presentes frecuentemente, en la catarata congénita, en el retinoblastoma y en la retinopatía de la prematuridad. El objetivo fue evaluar, en consulta con el oftalmólogo, el resultado de la evaluación a través del examen del reflejo rojo realizado por enfermeras, considerado "sospechoso" en un grupo de recién nacidos. Estudio descriptivo, realizado en una institución pública, en Fortaleza-CE, de noviembre/2005 a marzo/2006. El examen del reflejo rojo fue realizado por dos enfermeras, una alumna de enfermería a un grupo de 180 recién nacidos; 24 de estos presentaron matices del reflejo fuera del padrón, sobresaliendo amarillo con áreas más blanquecinas al centro o con presencia de rayas. Sin embargo, solamente fue posible encaminar a seis niños al oftalmólogo. Después de la fundoscopia, los hallazgos encontrados en el examen del reflejo rojo fueron comprobados, pero fueron considerados variaciones de la normalidad. Las intervenciones multiprofesionales favorecen el desarrollo de acciones eficaces destinadas a la prevención de la ceguera y a dar continuidad al proceso de atención de estos niños.

Palabras Claves: Salud ocular. Ceguera. Recién Nacido.

'Enfermeira, Doutora, Professora Adjunta do Departamento de Enfermagem da Universidade Federal do Ceará - UFC, Pós-Doutora pela Escola de Enfermagem da Universidade de Victoria/Canadá. Pesquisador 2 CNPq. Coordenadora da área Saúde Ocular da Criança do Projeto Saúde Ocular/UFC/ CNPq. Rua Ipê, 250. Apto 802 Torre II. São Gerardo - Fortaleza. Brasil. E-mail: cardoso@ufc.br, Enfermeira, Mestranda da Programa de Pós-Graduação em Enfermagem do Departamento de Enfermagem - UFC, Bolista FUNCAP, Membro do Projeto Saúde Ocular/ UFC/CNPq. Brasil. E- mail: adrianaufc@gmail.com, ${ }^{3}$ Enfermeira, Doutora, Professora Titular da Faculdade Integrada da Grande Fortaleza - FGF, Membro do Projeto Saúde Ocular, UFC/UFC/CNPq. Brasil. Email: ingrid@fgf.edu.br, ${ }^{4}$ Médica oftalmologista. Presidente da Sociedade Brasileira de Oftalmologia Pediátrica. Diretora Clínica do Centro de Aperfeiçoamento Visual Islane Verçosa. Oftalmologista do Hospital Geral de Fortaleza. Brasil. E-mail: islaneverc@gmail.com 


\section{INTRODUÇÃO}

0 sistema ocular, permeado por fatores neurológicos, genéticos e ambientais, evolui progressivamente durante toda a infância até atingir um estágio próprio de maturação. A perda da capacidade visual representa consequências adversas para o indivíduo e a sociedade, agravadas quando consideramos que a maior parte desses casos poderia ser evitada.

É preocupação da Organização Mundial de Saúde (OMS), há décadas, o estabelecimento de programas e de grupos de prevenção da cegueira, e, embora sejam muitos os esforços, ainda são restritos os recursos disponíveis. Também, constatamse dificuldades quanto à implementação de projetos preventivos em virtude do crescimento populacional, do aumento do número de indivíduos idosos (aumento da esperança de vida) e da carência de serviços oftalmológicos. ${ }^{1}$

A fim de revitalizar e fortalecer programas e grupos existentes, bem como criar novos grupos, a OMS propôs 0 programa "VISION 2020: The Right to Sight". Trata-se de esforço de colaboração entre a OMS e diversos parceiros organizações governamentais e não governamentais, grupos e instituições - que apresenta o objetivo de eliminar a cegueira evitável por volta do ano 2020.2

Sendo a cegueira um importante problema de saúde pública, a prevenção é o melhor caminho para assegurar a visão e a consequente qualidade de vida das crianças. Aproximadamente $50 \%$ das causas de cegueira infantil no Brasil poderiam ser prevenidas, como a rubéola e a toxoplasmose, ou tratáveis, como a retinopatia da prematuridade, catarata e glaucoma. ${ }^{2}$

A etiologia da cegueira em crianças e sua prevalência diferem, ao longo do tempo, e variam amplamente de região para região, sendo alguns dos fatores determinantes o nível de desenvolvimento socioeconômico e a disponibilização de serviços primários de saúde. Em países de baixa renda, a prevalência pode ser em torno de 1,2/1.000 crianças, enquanto em países desenvolvidos a prevalência é de cerca de $0,3 / 1.000$. $^{3}$

Para a elaboração e implementação de aç̃̃es eficazes na área da saúde ocular da criança, prevê-se a necessidade de contar com uma série de profissionais, oftalmologistas, outros médicos, educadores de saúde, administradores, enfermeiros, professores, assistentes sociais, terapeutas ocupacionais, entre outros, respeitadas as especificidades profissionais e condições locais de atuação. ${ }^{4}$

A identificação e o tratamento precoces de alterações oculares na infância constituem uma das cinco prioridades dos programas de prevenção da cegueira. Além disso, muitas condições associadas à cegueira na infância são causas de mortalidade infantil, como o sarampo, a hipovitaminose A, a rubéola congênita e a prematuridade. ${ }^{4}$

A prevenção e a detecção de alterações visuais no desenvolvimento infantil são práticas que devem ser mais valorizadas no Brasil. No caso das deficiências sensoriais, essa preocupação justifica-se pela possibilidade de antecipação do processo de intervenção logo no início da vida da criança, garantindo a estimulação necessária em todos os aspectos fundamentais para seu desenvolvimento global. Nessa área, os procedimentos de triagem são utilizados por se caracterizarem como instrumentos de baixo custo, de simples aplicação e eficientes. $^{5}$

Nessa perspectiva, o Teste do Reflexo Vermelho (TRV), incorporado à rotina de cuidados do recém-nascido, possibilita a detecção precoce de patologias oculares, que tem como sinal clínico a leucocoria, presente frequentemente na catarata congênita e no retinoblastoma. Trata-se de um importante teste de triagem, indicado para crianças em qualquer idade, podendo ser realizado ainda no berçário antes da alta hospitalar, pelo médico neonatologista ou enfermeira treinada em saúde ocular. ${ }^{6}$

A realização do teste já constitui rotina obrigatória em algumas localidades dos estados do Rio de Janeiro, São Paulo, Minas Gerais e Santa Catarina, regulamentada por meio de Projetos de Lei. ${ }^{6-7}$ Esse exame, também conhecido como "teste do olhinho", é rápido, simples e realizado com o auxílio de um oftalmoscópio direto, preferencialmente com o ambiente em penumbra.

0 objetivo do teste do reflexo vermelho não é a visualização das estruturas da retina (vasos, disco óptico e mácula), o que é feito pela fundoscopia, e sim avaliar a qualidade dos meios transparentes do olho (córnea, cristalino e vítreo). Portanto, quando se identificam opacificações nesses meios transparentes, o reflexo apresenta mudança em sua cor, o que justifica o encaminhamento para o profissional oftalmologista, ${ }^{9}$ o que se configura como uma ação importante do enfermeiro como agente ativo no processo de prevenção e tratamento das alterações visuais na clientela avaliada.

Embora o TRV se apresente como um exame simples, é necessária atenção durante sua realização, pois o examinador precisa verificar cautelosamente as características do teste e também considerar na avaliação variáveis que vão desde as condições de nascimento à integridade dos recursos materiais, ambientais e humanos. A classificação adotada pela Academia Americana de Pediatra utiliza os termos "normal ou presente", "anormal ou ausente". ${ }^{10}$ Em estudos realizados no Ceará, por enfermeiros em parceria com oftalmologista, têm-se utilizado os termos: normal, alterado e suspeito, para o resultado, e, além disso, a seguinte observação "presença ou ausência de leucocoria"." 11

0 resultado deste teste pode ser considerado negativo ou normal, quando o reflexo em ambos os olhos são equivalentes na cor, intensidade e clareza e não existem opacidades ou pontos brancos em alguma área, em um ou em ambos os reflexos observados. 0 resultado positivo ou anormal caracteriza-se de maneira oposta ao que foi mencionado. ${ }^{10}$

0 enfermeiro ou o pediatra, ao caracterizar o resultado do TRV como suspeito, deve encaminhar a criança para avaliação do oftalmologista, para que este possa realizar uma avaliação mais acurada utilizando outros recursos, para que se confirme 
Recém-nascidos com reflexo vermelho "suspeito" Cardoso MVLML, Aguiar ASC, Lúcio IML, Verçosa IC

ou não o resultado dado anteriormente. Entende-se o que o resultado do TRV considerado "suspeito" não deve ter uma classificação imediata como anormal, pois não identificou-se a leucocoria, achado clínico que caracteriza o reflexo vermelho como "alterado", mas apenas nuances que se distanciavam do laranja e vermelho.

Nos últimos quatro anos, por meio de atividade de pesquisa e ensino, tem-se dedicado com afinco a trabalhos direcionados para a saúde ocular do recém-nascido, na instituição onde o presente estudo foi realizado. Apesar de sua importância, o TRV ainda não se evidencia como rotina na assistência neonatal pelos profissionais de saúde, assim como a investigação de fatores da história materna e neonatal que podem manter relação com alterações visuais e o reflexo vermelho, embora alguns pais, e especialmente a mãe, já o conheça pelos meios de comunicação, como os telejornais.

Esse cuidado também foi incorporado ao longo desses três últimos anos, com uma abordagem multiprofissional, após um workshop realizado em Fortaleza, Ceará, em 2003-2004, organizado para compartilhar a abordagem da investigação da Retinopatia da Prematuridade - ROP, com profissionais de saúde que atendem a demanda de crianças com definição tardia de diagnóstico, assim como destacar o impacto de sua incidência, a relação com a assistência neonatal prestada e a correlação com os casos de cegueira na infância. Enfatizou-se a importância da triagem de recém-nascidos de risco para ROP, uma vez que estágios avançados da doença podem indicar o resultado do teste do reflexo vermelho como alterado.

Foram avaliados 180 recém-nascidos por meio do TRV em uma maternidade pública, em um estudo em 2007, quando se verificaram 24 resultados suspeitos. ${ }^{11}$ Esses resultados explicitam a relevância do tema, e, com isso, prosseguiu-se no processo de cuidado a essas crianças, encaminhando-as ao oftalmologista para avaliação especializada. Buscou-se, nessa estratégia de cuidado, a complementaridade de ações, visto que é necessária a coparticipação entre profissionais de saúde na perspectiva da prevenção e redução dos casos cegueira e promoção da saúde ocular da criança. Com base nas considerações, se questionou: 0 resultado do TRV em recémnascidos considerado suspeito pelo enfermeiro deve ser reavaliado pelo oftalmologista para que se confirme ou não 0 achado, em uma visão de complementaridade de ações interdisciplinares?

\section{OBJETIVO}

Avaliar, em consulta pelo oftalmologista, o resultado da avaliação pelo teste do reflexo vermelho realizado por enfermeiras e considerado "suspeito" em um grupo de recémnascidos.

\section{METODOLOGIA}

Estudo descritivo, quantitativo, desenvolvido no Projeto Saúde Ocular/UFC/ CNPq, área Saúde Ocular da Criança,
Esc Anna Nery Rev Enferm 2010 jan-mar; 14 (1): 120-25

realizado em uma instituição pública de referência (hospitalmaternidade) para o município de Fortaleza no estado do Ceará, no período de novembro de 2005 a março de 2006.

Duas enfermeiras treinadas em saúde ocular e uma bolsista CNPq, atuantes na área, avaliaram,. por meio do Teste do Reflexo Vermelho, 180 recém-nascidos independentes do peso, idade gestacional e condições de nascimento, que se encontravam internados na Unidade Neonatal (baixo e médio risco), Enfermaria Mãe - Canguru e Alojamento Conjunto. Aqueles que se encontravam em estado crítico de saúde, ou seja, em condições que implicassem instabilidade clínica e restrição ao manuseio, não fizeram parte do estudo.

Para a realização do teste, utilizou-se um oftalmoscópio monocular direto, observando-se os seguintes aspectos: sala com ambiente em penumbra para proporcionar a dilatação fisiológica das pupilas; manuseio do oftalmoscópio com lentes ajustadas no zero ou +2 a +5 , de acordo com a correção óptica do examinador; avaliação das condições do recémnascido, posicionando-o corretamente, de modo a favorecer o alinhamento de seu eixo visual; direcionamento do feixe de luz a uma distância de cerca de 40 a $50 \mathrm{~cm}$ dos olhos da criança e consequente observação do reflexo através do espaço da pupila. 0 teste foi classificado como: normal (ausência de leucocoria), alterado (presença de leucocoria) e suspeito. A análise dos dados se pautou na literatura específica e é apresentada de forma descritiva em números absolutos e percentuais.

Os recém-nascidos cujo resultado do teste foi classificado como "suspeito" ao final do estudo foram encaminhados para avaliação oftalmológica, mesmo aqueles residentes em localidades no interior do Estado. 0 estudo atendeu as considerações éticas dispostas na resolução 196/86 do Conselho Nacional de Saúde, tendo sido aprovado pela Comissão de Ética da instituição na qual foi realizado, mediante o ofício n⿳0 596/ 05 e protocolo no 263/05.

\section{RESULTADOS E DISCUSSÃO}

A partir da amostra estudada de 180 recém-nascidos, 24 deles tiveram o resultado do teste do reflexo vermelho classificado como "suspeito". Um terço deles (8) estavam inclusos em acompanhamento pelo Programa de Triagem para Retinopatia da Prematuridade, constando, inclusive, o registro da realização da fundoscopia realizada pelo profissional oftalmologista. Dentre os resultados dessa investigação diagnóstica, cinco recém-nascidos apresentavam retinopatia da prematuridade, em grau III, dois ainda se encontravam no estágio de retina avascular, e em um o achado mostrou pontos hemorrágicos na retina.

Logo, os demais recém-nascidos (16) foram encaminhados para a efetivação da consulta oftalmológica, mediante contato com os pais/responsáveis pela criança, através de telefone e endereços fornecidos; no entanto, foi possível apenas o contato com 12 deles. Destes, apenas seis compareceram ao exame realizado no consultório da oftalmologista, mediante auxílio 
transporte pelos integrantes do projeto Saúde Ocular/UFC/ CNPq, considerando-se a dificuldade referida em relação ao deslocamento, no dia 6 de maio de 2006.

\section{Perfil dos recém-nascidos e suas mães}

Caracterizou-se a história materna e neonatal dos 24 bebês que apresentaram resultado suspeito do reflexo vermelho, pois fatores importantes podem ser identificados e ter relação com alterações apresentadas pelo RN.

0 perfil encontrado para as mães mostrou que a idade materna variou de 15 a 39 anos, sendo que $4(16,6 \%)$ tinham idade inferior a 18 anos. Quanto ao estado civil, 6 eram mães solteiras, 4 casadas, e 14 informaram viver em união consensual. Ao analisar os registros referentes ao número de consultas de pré-natal, verificou-se que $10(41,6 \%)$ correspondiam a um número inferior a seis, sendo que o Ministério da Saúde ${ }^{12}$ recomenda que o mínimo seja de seis consultas. Seis (25\%) tinham história de vícios (tabagismo e/ou etilismo). Em estudo realizado com 37 mulheres que tiveram bebês prematuros, 0 número de consultas de pré-natal também evidenciou que 25 $(67,5 \%)$ realizaram entre 2 e 5 consultas, mostrando que também não atingiram o mínimo de seis consultas ${ }^{13}$.

Três (12,5\%) mães apresentaram infecção urinária durante o pré-natal e $5(20,8 \%)$ desenvolveram Doença Hipertensiva Específica da Gravidez (DHEG); 2 (8,3\%) amniorrexe prematura; 1 (4,1\%) "descolamento" prematuro de placenta. Verificou-se também que uma das mães tinha sífilis e 2 (8,3\%) apresentaram lgG (anticorpos) para rubéola e toxoplasmose. Além disso, 8 (33,3\%) tinham história familiar ou pessoal de erros de refração, $1(4,1 \%)$ tinha caso de estrabismo na família e $10(41,6 \%)$ de catarata.

Os fatores que se sobressaíram na história materna apresentaram relação com a prematuridade, considerado fatorchave para se investigarem alterações visuais em RN. Em geral, as infecções congênitas resultantes de agentes virais e não virais são chamadas TORCH - um acrônimo para toxoplasmose, outras infecções, rubéola, citomegalovírus e herpes. Essas patologias podem causar uma ampla faixa de sequelas, desde aborto espontâneo e morte fetal até infecção explícita ou assintomática ao nascer. ${ }^{14}$

As principais infecções congênitas que favorecem ao acometimento oftalmológico são: a toxoplasmose, a rubéola e o citomegalovírus. A toxoplasmose é uma importante causa de baixa de visão em crianças, podendo ocasionar, se não tratadas durante o primeiro ano de vida, lesões coriorretinianas. A infecção por citomegalovírus pode levar a cegueira, e aproximadamente $40 \%$ das mães com infecção primária transmitem para o feto, porém somente $10 \%$ a $15 \%$ têm a doença sintomática ao nascimento. ${ }^{9}$

A literatura comenta que a sífilis não tratada pode afetar a gestação, resultando em abortamentos, natimortos, trabalho de parto e nascimento prematuros, além de que pode causar anomalias oculares. A prevenção, durante a gravidez, inicia com o acesso precoce e contínuo do acompanhamento prénatal. ${ }^{10}$
No Brasil, a rubéola é um dos maiores responsáveis por casos de cegueira em RN. Adquirida nos três primeiros meses de gestação, pode causar malformação, glaucoma e catarata congênita no RN, o que justifica o cumprimento do programa de vacinação específico. ${ }^{3}$

Considerou-se relevante mencionar as intercorrências apresentadas pela mãe no pré-natal, pois estas, em sua maioria, contribuem para o parto prematuro que carreia uma imaturidade geral por parte do RN, podendo levar a uma disfunção em qualquer órgão ou sistema, inclusive o visual. ${ }^{15}$

Com relação às características da história neonatal, observou-se que 14 (58,3\%) nasceram por parto normal, 10 $(41,6 \%)$, por cesariana. Quatorze $(58,3 \%)$ nasceram com idade gestacional inferior a 37 semanas, portanto considerados RN pré-termos, e os outros $10(41,6 \%)$ nasceram a termo (entre 37 e 42 semanas de gestação); 13 (54,1\%) deles eram do sexo feminino e 11 (45,8\%), do masculino. 0 peso dos recém-nascidos variou de 715 a 3.650 gramas, sendo que $15(62,5 \%)$ deles foram classificados como de baixo peso (inferior a 2.500 gramas). Correlacionando peso e idade gestacional, verificou-se que 9 (37,5\%) RN foram considerados PIG - Pequenos para a Idade Gestacional, e 15 (62,5\%), AIG - Adequados para a Idade Gestacional.

Os escores do APGAR variaram no primeiro minuto de 3 a 8 e no quinto minuto de 7 a 9; $13(54,1 \%)$ bebês foram submetidos a fototerapia e $16(66,6 \%)$ a oxigenoterapia. Dos que necessitaram de oxigenoterapia, 4 (25,0\%) foram tratados apenas por meio da modalidade de HOOD (oxigenação através de halo/capacete); 6 (37,5\%) por HOOD e CPAP (ventilação por pressão positiva contínua nas vias aéreas) e os demais, 6 (37,5\%) por H0OD, CPAP e VM (ventilação mecânica). 0 tempo de exposição ao oxigênio variou de 6 a 32 dias. Dos recémnascidos, 5 (20,8\%) fizeram transfusão sanguínea.

Quanto à história neonatal apresentada, notou-se uma quantidade maior de recém-nascidos prematuros. É comentado na literatura que o nascimento antes da gestação a termo aumenta bastante o risco de problemas neonatais, tais como 0 comprometimento do desenvolvimento pelos efeitos do tratamento médico intensivo, como também aqueles ocasionados por sobrecarga sensorial e estresse ambiental. Do mesmo modo, torna-se grande a probabilidade de a mãe desvincular-se emocionalmente da criança. ${ }^{14}$

0 risco para apresentar ou desenvolver uma alteração visual é secundário a essa imaturidade orgânica, de estruturas que ainda não atingiram a maturidade funcional ao nascimento Como visto anteriormente, a maioria dos RN fez uso de oxigenoterapia, como a ventilação mecânica, passando ao CPAP nasal até o desmame pelo oxi-hood. A saturação sanguínea de oxigênio e a concentração de oxigênio administradas devem ser monitorizadas para evitar a administração desnecessária ou de altas concentrações por tempo prolongado, que poderão causar efeitos tóxicos na retina e nos pulmões, principalmente de RN prematuros. ${ }^{15}$ 
Recém-nascidos com reflexo vermelho "suspeito" Cardoso MVLML, Aguiar ASC, Lúcio IML, Verçosa IC

Quanto à fototerapia, 13 bebês a utilizaram. Destaca-se um cuidado importante, que é a colocação adequada do protetor ocular, pois a incidência da luz pode ocasionar degeneração da retina. ${ }^{16}$

Em um estudo no qual se realizou uma avaliação visual sistemática em uma amostra de 114 RN, identificou-se 13 (11\%) RN com o reflexo vermelho suspeito. Foram associadas algumas variáveis da história neonatal, encontrando-se associações estatísticas significantes entre reflexo vermelho e Apgar no primeiro minuto de vida $(p=0,041)$ e o tempo de oxigenoterapia $(p=0,028)$. As condutas de enfermagem, a partir dos achados da avaliação visual, foram dirigidas ao prontuário, à equipe de enfermagem, aos cuidados com a visão do recém-nascido ao utilizar terapêuticas específicas, aos seus pais e a outros profissionais (encaminhamento). ${ }^{15}$

\section{Avaliação oftalmológica}

Dos recém-nascidos (RN) que apresentaram reflexo vermelho suspeito, além da relevância das suas histórias de vida e condições de saúde, percebeu-se pelas enfermeiras uma coloração do reflexo diferente ao que geralmente é descrito na literatura, sendo que a maioria apresentou o reflexo mais para o amarelo com manchas mais claras (esbranquiçadas) ao centro ou presença de "rajadas". Em relação à intensidade, algumas vezes observou-se diferença entre os olhos, motivos que as levaram a classificar o resultado como "suspeito" e a encaminhar esses RN para esclarecimento oftalmológico.

A avaliação pela oftalmologista dos RN triados e que apresentaram teste do reflexo vermelho "suspeito" representou aspecto importante para o estudo. Em consulta de seguimento, foram realizados fundoscopia e mapeamento de retina.

Segundo a Sociedade Brasileira de Oftalmologia, o mapeamento de retina ou oftalmoscopia indireta permite a avaliação de toda a superfície da retina, em particular da região periférica, que não pode ser observada pela oftalmoscopia convencional, pois esta permite a avaliação apenas do pólo posterior da retina. Esse exame avalia as estruturas internas dos olhos (retina, nervo óptico e vasos), sendo muito útil no diagnóstico e caracterização do descolamento de retina, da retinopatia diabética, das uveítes e de diversas retinopatias. ${ }^{17}$

Todas as crianças avaliadas apresentaram, ao exame de fundo de olho, papilas normocoradas, região macular, vasos e periferia sem anormalidades e ausência de leucocoria. Algumas apresentaram fundustigroide, outros fundus empalidecido. Uma criança mostrou ao exame tortuosidade arteriolar discreta, porém sem significado clínico. Em outra, houve suspeita de ametropia-miopia, e sua mãe foi orientada a procurar 0 ambulatório de oftalmologia quando a criança completasse cerca de 1 ano e meio de idade.

Essa fase representa um marco importante para o processo de amadurecimento orgânico e estabilidade funcional da visão, pois esta se desenvolve $90 \%$ durante os dois primeiros anos de vida. Portanto, é durante esta fase que a criança aprende a
Esc Anna Nery Rev Enferm 2010 jan-mar; 14 (1): 120-25

fixar, a movimentar os olhos de maneira conjunta e a perceber profundidade. Os outros $10 \%$ do desenvolvimento visual ocorrem entre 7 e 9 anos de idade. A Sociedade Brasileira de Oftalmologia Pediatra recomenda um exame a cada 6 meses nos dois primeiros anos de vida e, depois, se tudo normal, um exame anual até os 8 ou 9 anos. $^{17}$

Os achados visuais relativos à realização do teste do reflexo vermelho em recém-nascidos neste estudo foram confirmados e mais bem detalhados pela avaliação oftalmológica. Aspectos outrora duvidosos para as autoras em relação à percepção da cor do reflexo, ao serem visualizados pelo oftalmoscópio, também foram discutidos com a oftalmologista, destacando-se aqueles relativos a variações na normalidade, ou seja, no desenvolvimento da retina normal, a qual se apresenta em processo de vascularização, como se observa em recémnascidos.

Estas considerações, processadas entre médica e enfermeiras foram imprescindíveis para a orientação da prática do teste do reflexo vermelho, em especial com recém-nascidos, pois a avaliação visual é abordada na formação do enfermeiro ainda de modo restrito. Após a realização da consulta oftalmológica especializada, discutiu-se acerca da importância e necessidade do cuidado multiprofissional, quando se trata de prevenção à cegueira.

Sendo assim, verifica-se a importância das organizações educacionais, formadoras desses profissionais, que alertem para a inclusão, em seus currículos, de assuntos que enfoquem a assistência ao cliente com alteração/deficiência visual. ${ }^{18}$

Neste estudo, a relação médico-enfermeiro visou o bemestar, a promoção, a prevenção e a recuperação da saúde de crianças em idade tenra. Discutiu-se também acerca das variações da normalidade relativas à retina, tendo sido considerações relevantes por se tratar de um teste ainda pouco utilizado na prática clínica, tanto por médicos como por enfermeiros, e em população que apresenta peculiaridades na avaliação visual, recém-nascidos.

\section{CONCLUSÃO}

Introduzir questões envolvendo o cuidado em saúde ocular no período neonatal tem sido um desafio, assim como sensibilizar e preparar os profissionais para a necessidade de triagens, dentre essas a visual, além da realização sistemática da avaliação visual do recém-nascido no cuidado prestado.

No que abrange esse cuidado, médicos e enfermeiros têm importante papel com vistas a minimizar agravos à saúde do recém-nascido, auxiliando na identificação precoce de alterações visuais e encaminhando essas crianças para investigação diagnóstica especializada, com o oftalmologista. Os resultados do estudo demonstram a importância da integração da equipe de saúde, visto que as crianças que apresentaram teste do reflexo vermelho suspeito pelos enfermeiros puderam ser reavaliadas em uma visão mais aprofundada de recursos tecnológicos pelo oftalmologista, e 
Recém-nascidos com reflexo vermelho "suspeito" Cardoso MVLML, Aguiar ASC, Lúcio IML, Verçosa IC

isso permitiu uma relação harmoniosa. Todos os resultados suspeitos foram considerados normais, mesmo que as mesmas características identificadas tenham sido, também, observadas pelo especialista.

Desse modo, principalmente por meio de ações simples de baixo custo, como a utilização do teste do reflexo vermelho, no intuito de identificação precoce de leucocorias, o enfermeiro

\section{REFERÊNCIAS}

1. World Health Organization-WHO. Strategies for the prevention of blindness in national programmes; a primary healthy care approach. $2^{\text {nd }}$ ed. Geneva; 1997. [citado 05 abr 2007]. Disponível em: http:// www.v2020.org/page.asp.

2. Ventura LM, Travassos S, Ventura AG, Trigueiro L, Marques S. Um programa baseado na triagem visual de recém-nascidos em maternidades: Fundação Altino Ventura-2000. Arq Bras Oftalmol 2002; 65: 628-35.

3. Temporini ER, Kara-José N. A perda da visão: estratégias de prevenção. Arq Bras Oftalmol 2004; 67(4): 597-601.

4. Liang L. Estratégias para prevenção da cegueira infantil. [on-line] São Paulo (SP): Universo Visual; 2006. [citado 11 set 2006]. Disponível em: URL:http://www.universovisual.com.br/publisher/preview.php.

5. Lima MCMP, Barbarini GCO, Gagliardo HGRG, Arnais, MAO, Gonçalves VMG. Observação do desenvolvimento de linguagem e função auditiva e visual em lactentes. Rev Saude Publica 2004; 38(1): 106 -12.

6. Projeto Luz. [on-line] [citado 19 set 2005]. Disponível em: URL:http:/ /www.unigranrio.br/medicina/projprojetoluz.html.

7. Wasilewski D, Zago RJ, Bardal AMC, Heusi TM, Carvalho FP, Maciel LF, et al. Importância da avaliação oftalmológica em recém-natos. J Pediatr 2002; 78(3).

8. Reis PP. Reflexo vermelho: textos científicos. Belo Horizonte (MG): Sociedade Mineira de Pediatria; 2005.

9. Graziano RM. Exame oftalmológico do recém-nascido no berçário: uma rotina necessária. J Pediatr 2002; 78(3): 187-88. tem condições de atuar, em uma visão interdisciplinar, na prevenção dos casos de cegueira na infância e na promoção da qualidade vida. A relação médico-enfermeira foi essencial para assegurar o seguimento das crianças em relação aos achados observados e considerados relevantes, tanto à avaliação efetiva quanto à prática do teste do reflexo vermelho, o qual, no contexto do cuidado de enfermagem, ainda é recente.

10. American Academy of Pediatrics. Section Ophthalmology. Red reflex examination in infants. Pediatr 2002; 109(5): 980-81.

11. Aguiar, ASC, Cardoso, MVLML, Lúcio IML. Teste do reflexo vermelho: forma de prevenção à cegueira na infância. Rev Bras Enferm 2007; 60(5): 541-45.

12. Ministério da Saúde (BR). Manual técnico de pré-natal e puerpério: atenção qualificada e humanizada. Brasília (DF); 2005.

13. Santos FLB, Oliveira MIV, Bezerra MGA. Prematuridade entre recémnascidos de mães com amniorrex prematura. Esc Anna Nery Rev Enferm 2006 dez; 10(3): 432-38.

14. Kenner C. Enfermagem neonatal. $2^{a}$. ed. Rio de Janeiro(RJ): Reichmann \& Affonso; 2001.

15. Lucio IML, Cardoso MVLML, Almeida PC. Investigation of the related factors between newborn history and red reflex. Rev Esc Enferm 2007; 41(2).

16. Moreira MEL, Lopes JMA, Carvalho M, et al. 0 recém-nascido de alto risco: teoria e práticas do cuidado. Rio de Janeiro (RJ): Fiocruz; 2004.

17. Sociedade Brasileira de Oftalmologia. [on-line] [citado 03 nov 2008]. Disponível em: http:// www.sboportal.org.br.

18. Favretto DO, Carvalho EC, Canini SRMS. Intervenções realizadas pelo enfermeiro para melhorar a comunicação com deficientes visuais. Rev Rene 2008 jul/set; 9(3): 68-73. 